\title{
Using Scanning Electron Microscopy and Atomic Force Microscopy to Study the Formation of Nanoparticles on Red Blood Cell Surface in Cervical Cancer Patients
}

Sargylana N. Mamaeva, $\mathrm{PhD}^{1}$; Irina V. Kononova, $\mathrm{PhD}^{2 *}$; Michael Ruzhansky, $\mathrm{PhD}^{3,4}$; Petr V. Nikiforov ${ }^{2,5}$; Nadezhda A. Nikolaeva ${ }^{1}$; Alexandr N. Pavlov ${ }^{1}$; Nyurguyna F. Fedorova ${ }^{1}$;

Junqing Huang; ${ }^{3}$ Motrena N. Semenova ${ }^{1}$; Daiaana V. Barashkova ${ }^{1}$; Lyubov S. Frolova ${ }^{6}$; Georgy V. Maksimov, $\mathrm{PhD}^{7,8}$

\author{
${ }^{1}$ M.K. Ammosov North-Eastern Federal University, Medical Institute, Yakutsk, Russia \\ ${ }^{2}$ Yakut Scientific Center of Complex Medical Problems, Yakutsk, Russia \\ ${ }^{3}$ Ghent University, Belgium \\ ${ }^{4}$ Queen Mary University of London, the United Kingdom \\ ${ }^{5}$ Yakut Republican Oncology Center, Yakutsk, Russia \\ ${ }^{6}$ Lomonosov Moscow State University-PPI Shenzhen (Shenzhen, China) \\ ${ }^{7}$ Lomonosov Moscow State University, Moscow, Russia \\ ${ }^{8}$ National University of Science and Technology "MISiS”, Moscow, Russia
}

\begin{abstract}
Background: In this study, we used scanning electron microscopy (SEM) and atomic force microscopy (AFM) to examine the changes in morphology of red blood cells (RBCs) and to investigate the nanoparticles (NPs) found on their surface in cervical cancer (CC) patients undergoing radiation therapy (RT).

Methods and Results: We obtained smears of venous blood from $12 \mathrm{CC}$ patients at the start, midway and at the end of external beam RT and then midway and at the end of brachytherapy. It was found that in CC patients, the number of RBCs with abnormal morphology increased and NPs appeared on their surface. During RT, the total number of abnormally shaped RBCs and the number and size of NPs increased. The RBC diameter was $8.38 \pm 0.36 \mu \mathrm{m}$ in the control group and $9.41 \pm 0.47 \mu \mathrm{m}$ in CC patients. The average diameter of NPs on the RBC surface was $69.91 \pm 12.15 \mathrm{~nm}$ and their average height $23.75 \pm 3.70 \mathrm{~nm}$. After RT, the morphology of RBCs was restored, and the formation of NPs decreased.

Conclusion: The changes observed could serve as the basis for developing efficacy indicators of cancer radiation therapy. (International Journal of Biomedicine. 2020;10(1):70-75.)
\end{abstract}

Key Words: extracellular vesicle $\bullet$ red blood cell $\bullet$ nanoscale $\bullet$ morphology $\bullet$ radiation therapy

\section{Abbreviations}

AFM, atomic force microscopy; CC, cervical cancer; EV, extracellular vesicle; HPV, human papillomavirus; NP, nanoparticle; REM, reflection electron microscopy; RT, radiation therapy; RBC, red blood cell; SEM, scanning electron microscopy

\section{Introduction}

Every year approximately 500,000 women are diagnosed with CC. ${ }^{(1)}$ It is the second most common cancer among women and is very deadly, accounting for around 260,000 deaths worldwide, ${ }^{(2)}$ making it the leading cause of death by cancer among the female population of developing countries. ${ }^{(3)}$ 
While there is overwhelming evidence to suggest that persistent HPV infection is the key reason for the development of cervical carcinoma, other factors also contribute to the establishment and progression of cancer. Several studies have shown elevated levels of EVs in the bodily fluids of cancer patients, ${ }^{(4)}$ including CC patients specifically. ${ }^{(5)} \mathrm{EVs}$ can be broadly defined as membrane particles released into the extracellular space by a cell of any type. ${ }^{(6)}$ EVs typically range from $30 \mathrm{~nm}$ to $2,000 \mathrm{~nm}$ in diameter ${ }^{(7)}$ and can be broadly classified into exosomes, microvesicles and apoptotic bodies, according to their biogenesis. ${ }^{(7,8)}$ While microvesicles and apoptotic bodies arise through direct blebbing off the membrane of a normal or apoptotic cell, respectively, exosomes are generated through the endolysosomal pathway. ${ }^{(7)}$ Existing research recognises that an increased abundance of exosomes in cancer patients' blood ${ }^{(9,10)}$ may play a role in the so-called cancer "field effect," in which cancer cells induce malignant changes in the surrounding healthy cells. ${ }^{(11)}$ In fact, there is a large body of research dedicated to the role that exosomes play in cell-to-cell communication by carrying molecular messages, often in the form of miRNA, from the parent cell to the target cell. . $^{(5,12,13)}$

RT, which is a common treatment type for cervical carcinoma, may exacerbate the cancer field effect as irradiation has been shown to produce non-targeted effects in the cells that have not themselves been irradiated. ${ }^{(14)}$ This can, at least partially, be attributed to the stimulating effect radiation has been shown to have on the secretion of exosomes and their subsequent uptake by the surrounding cells. ${ }^{(14-17)}$

While there is a wealth of studies dedicated to tumorderived exosomes in the bloodstream of oncology patients, ${ }^{(9,10)}$ it appears that cancer cells may not be the only perpetrators of elevated EV levels in cancer sufferers. In fact, a study by Kim et al.(2003) has demonstrated that the quantity of platelet MVs in gastric cancer patients' blood was more than 3 times higher than in healthy controls, with platelet MV quantities of $>2.70 \times 10^{9} / \mathrm{ml}$ correlating with the presence of distant metastasis and poor prognosis. ${ }^{(4)}$ This indicates a need for a more complex understanding of various blood parameters, including EV abundance, as biomarkers of cancer progression. Moreover, the current focus of scientific inquiry rests on freely circulating EVs or their uptake by the surrounding cells; however, a recent study has found that roughly two thirds of the total blood EV count in breast cancer patients exists in the blood cell-bound state. ${ }^{(18)}$ A thorough investigation of blood cell-bound EVs as potential cancer biomarkers is therefore entirely warranted.

In the present study, we use SEM and AFM to examine the changes in morphology of RBCs and to investigate the nanoscale objects found on their surface in CC patients undergoing RT. While it is beyond the scope of the current study to identify the precise biogenesis of these objects, we propose that they could be cell-bound EVs of various origins.

\section{Materials and Methods}

\section{Blood Samples}

Smears of venous blood containing K3-EDTA from 12 patients with $\mathrm{CC}$ and from 3 patients of the control group were obtained. The age range was from 45 to 55 years. For $4 \mathrm{CC}$ patients, analyses were taken at each stage of the full course of treatment, and for the remaining $8 \mathrm{CC}$ patients, right after the first stage. A thin, even layer of blood was smeared onto a clean degreased glass slide and dried.

\section{$\underline{\text { SEM and AFM imaging }}$}

SEM and AFM were used to investigate the morphology and surface of RBCs in CC patients at the start, midway and at the end of external beam radiation therapy and then midway and at the end of brachytherapy. A high-resolution SEM JSM7800F (Japanese Electron Optics Laboratory, JEOL, Japan) equipped with a Schottky thermal field emission cathode and a super hybrid objective lens was used. The microscope is equipped with a Gentle Beam system, which reduces the speed of electron propagation of the emission beam and the acceleration of emitted electrons, which significantly increases the signal-to-noise ratio and image quality at low accelerating voltages. The following microscope parameters make it possible to study the morphology of the RBC surface in blood smears without spraying conductive coatings, to eliminate damage to the object and to identify NPs (resolution from $0.08 \mathrm{~nm}$ to $1.2 \mathrm{~nm}$, magnification range $25-1000000 \mathrm{x}$, at voltage $15 \mathrm{kV}$ and $1 \mathrm{kV}$, respectively).

To investigate the morphology of RBCs, glass and graphene substrates and various SEM amplification modes were used. The studies were carried out at accelerating voltages of $1 \mathrm{kV}$ and $2 \mathrm{kV}$ with a voltage of $8-10 \mathrm{~V}$ applied to the test object. The maximum size of the samples was $20 \mathrm{~mm}$.

The size analysis of RBCs and NPs was carried out using the software JMicroVision v1.2.7 (Roduit, 2007). The data determined were entered in Excel and histograms of the sizes of RBCs and NPs were constructed using standard methods. Average values, dispersions, standard deviations, and size distributions of RBCs and NPs were determined using the Gauss distribution.

AFM was performed using a Solver Next microscope (NTMDT company) with scanning area $50 \times 50 \mu \mathrm{m}$ (512 points) and $5 \times 5 \mu \mathrm{m}$ (512 points); scanning speed $0.5 \mathrm{~Hz}, 0.25 \mathrm{~Hz}$; NSG10 cantilever with a radius of curvature of not more than $10 \mathrm{~nm}$. The AFM images were recorded using Nova Px and Image Analysis (NT-MDT company) software. The S1 ruler was used to assess the RBC surface unevenness and thickness.

\section{Radiation Treatment}

In the Yakut Republican Oncology Dispensary (Yakutsk, Russia), patients were treated with RT, which consists of two stages: first, distance RT using the Elekta Synergy accelerator (United Kingdom, external beam radiation therapy, 6-18 MeV) and then brachytherapy using the MultiSource HDR device (Germany, brachytherapy with Cobalt-60 source). At the first stage, the treatment was performed on a linear accelerator in the mode of working with electrons with energy of $6 \mathrm{MeV}$. The therapy was carried out as follows: 3 times for 5 days daily with a break of 2 days (i.e. at the first stage only 15 fractions of $2 \mathrm{~Gy}$ were carried out). Then, without a break between the first and second stage, contact RT of 5 Gy was performed, interspersed with distanced RT of 2 Gy of 5 fractions of each type of RT. 
Blood samples were collected at the beginning, middle and end of the remote therapy, then in the middle and at the end of the contact RT. Multiple methods (3D CRT, IMRT, VMAT) were employed in the course of therapy to ensure precision delivery of high doses to the tumor and low doses to healthy tissue.

\section{Primary methods for processing experimental results}

To determine the linear size of RBCs, REM images were used at magnification of 1000x, and for NP sizes, REM images were used at magnification from $30,000 \mathrm{x}$ to $50,000 \mathrm{x}$. Primary methods of statistical processing of experimental data were used to determine the linear sizes of RBCs and NPs. The diameters of RBCs and NPs were measured using the computer program JMicroVision 1.2.7, which is freely available. Using Excel, we constructed histograms of linear sizes of RBCs and NPs on the RBC surface in normal and pathological conditions for each patient; based on these histograms, the distribution of linear sizes was determined as a normal Gauss distribution. Then, based on a sample of the average size of RBCs and NPs of all patients using the Shapiro-Wilk test, the hypothesis of a normal distribution of linear sizes was tested. This criterion was used, taking into account that it is reliable for a test volume of $8 \leq n \leq 50$.

The study was conducted in accordance with ethical principles of the WMA Declaration of Helsinki (1964, ed. 2013) and approved by the Ethics Committee of the M.K. Ammosov North-Eastern Federal University (protocol No. 13 of April 4, 2018, decision No. 2). Written informed consent was obtained from each patient.

\section{Results and Discussion}

In the course of the present study, linear size distribution histograms for RBCs and NPs were obtained, and abnormally shaped RBCs, or poikilocytes were detected (Fig.1 a,b). An increased abundance of poikilocytes and NPs (Fig.1 c, d) was observed in CC patients. The RBC diameter was $8.38 \pm 0.36 \mu \mathrm{m}$ in the control group and $9.41 \pm 0.47 \mu \mathrm{m}$ in $\mathrm{CC}$ patients.

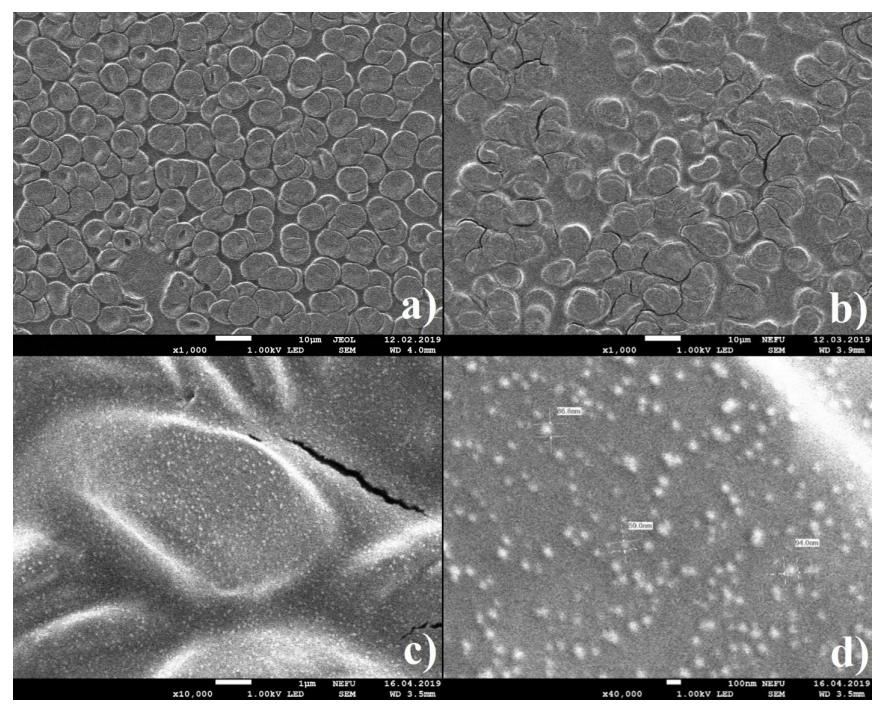

Fig. 1. SEM image of RBCs in patients with CC a) before RT; b) after RT, $\times 1000$; c) SEM images of a blood sample in case of $C C, \times 10000$, and d) $\times 40000$
In this paper, discussions of the results and the authors' conclusions were based on certain sizes of NPs on the RBC surface. Obviously, determining the size of these particles is a major and important part of the study. Accordingly, improving image quality is a prerequisite for the closest-to-reality identification of NPs. In the future, the authors plan to use the most accurate approaches to study the morphology of RBCs and NPs on their surface. One such approach is presented below by improving the contours of NPs on the RBC surface in the SEM image at a magnification of 100,000x. In some situations, these microscope images may suffer from noise. In our case, "pepperand-salt" noise and Gaussian noise occur frequently under the high-magnification, high-resolution imaging situations. In general, the size of the nanoparticles is around 10-50 pixels in $100,000 \mathrm{x}$, and that of noise can be $3-5$ pixels. In this case, it is always difficult to measure the size of nanoparticles with high accuracy. In order to reduce the interference of the noise, we introduced two denoising methods: a median filter and an L0norm smoothing filter. The median filter is proved to be helpful in removing the pepper-and-salt noise, while the L0-norm smoothing filter shows a strong ability to filter out the noise and to preserve the salient edges. The median filter is very easy. We considered a small local path centroid in pixel $k$, and vector $\boldsymbol{I}_{\boldsymbol{k}}=$ $\left(\boldsymbol{I}_{k, 1}, \boldsymbol{I}_{k, 2}, \boldsymbol{I}_{k, n}\right)$ to represent the values of all pixels in this patch. Then we sorted the $\boldsymbol{I}_{k, I}, \boldsymbol{I}_{k, 2}, \boldsymbol{I}_{k, n}$ in ascending order, and the filter output $\left.\overline{\boldsymbol{I}}_{\boldsymbol{k}}=\boldsymbol{I}_{\boldsymbol{k}} \mathrm{L}^{\mathrm{n} / 2}\right\lrcorner$, where $\left.{ }_{\llcorner} \mathbf{n} / \mathbf{2}\right\lrcorner$ is the largest natural number less than $\mathbf{n} / \mathbf{2}$. We set the patch size $w=5, \mathrm{n}=5 \times 5$. As shown in Figure 2 (in the middle of first row), the pepper-and-salt noise is significantly suppressed in comparison with the original image. Moreover, in order to compute the size of nanoparticles more accurately, we introduced the L0-norm smoothing filter to boost the edge. Mathematically, this method is based on an optimization framework and can preserve the main edges of the nanoparticles while smoothing the local small gradients. The reader is referred to the algorithm ${ }^{(19)}$ for more details. As shown in Figure 2 (top right), the outline of NPs is very clear now compared to the source image. It is easy to compute the size by counting the number of pixels. In Figure 2 (bottom), we also compared the results in 1D case. Obviously, the original data (blue) has strong pepper-and-salt noise, and the median filter (red) can reduce some noise but the outline of NPs is still not clear, while these residual noises can be further reduced with L0-norm filter (yellow).
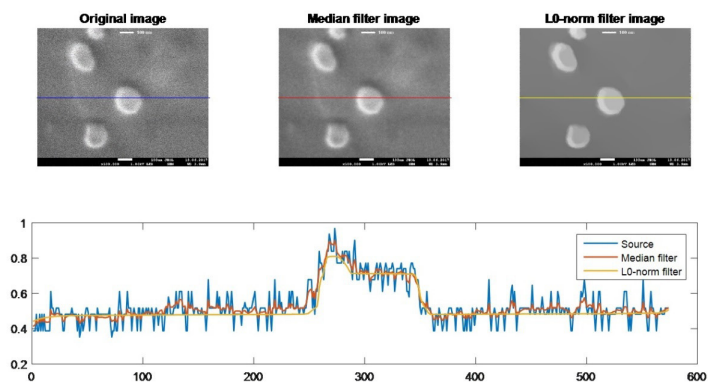

Fig. 2. Microscope Image Filtering Results

Ionising radiation was found to influence the size and morphology of RBCs. The overall quantity of poikilocytes 
increased from $19 \%$ to $23 \%$ in the course of RT with approximately a $1 \%$ increase at each stage of therapy. The ratio of poikilocytes also varied according to the RT stage. The number of ruptured RBCs increased from $11 \%$ to $24 \%$ while the number of discocytes decreased from $77 \%$ to $71 \%$. Drepanocytes-like cells (7\% midway through and 5\% at the end of RT) and echinocytes (12\% at the start of RT) were also detected.

Comparative analysis of the $\mathrm{RBC}$ size in the control group and the group with $\mathrm{CC}$ by mathematical statistics shows that the distribution of linear sizes of RBCs has the form of single peaks with the same width at half-height of the peaks shifted relative to each other. The average value of the linear size of the RBC smears of the control group was 6.91 microns. The average value of the RBC diameter of CC patients was 7.64 microns.

In the present study, the number of NPs (from $20 \mathrm{~nm}$ to $110 \mathrm{~nm}$ in diameter) in the blood of CC patients was increased (Fig.3a). The average diameter of the discovered objects was $69.91 \pm 12.15 \mathrm{~nm}$ and their average height $23.75 \pm 3.70 \mathrm{~nm}$. Changes in the size and quantity of NPs during RT were observed in the course of this study. The size range of NPs was $20 \mathrm{~nm}$ to $70 \mathrm{~nm}$ at the start of the treatment and $20 \mathrm{~nm}$ to $110 \mathrm{~nm}$ midway through the treatment. The quantity of NPs increased with each stage of RT from 136 at the start to 192 in the middle of the second stage of the treatment. Once RT was finished the number of NPs dropped (Fig. 3a).

SEM images before and after RT revealed $\mathrm{RBC}$ agglutination (Fig.1b), or "clumping" in all samples. Characteristic peaks, which are presumed to be NPs, were observed on the RBC surface on the AFM images (Fig. 4). The figure shows the linear dimensions of NPs on the RBC surface (Fig. 3 b,c).

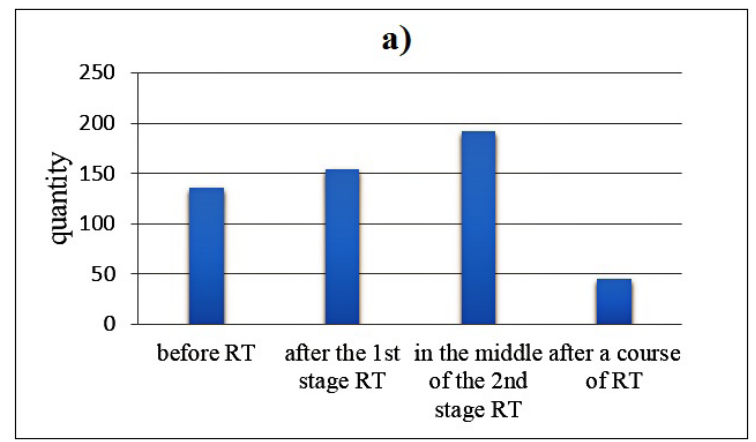

b)

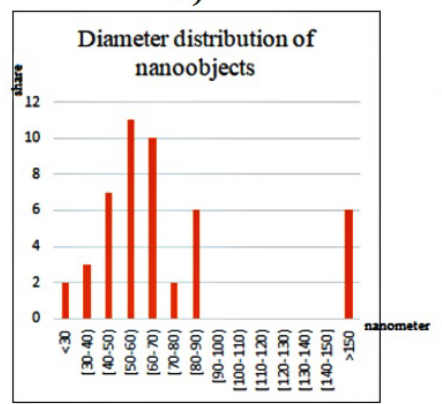

c)

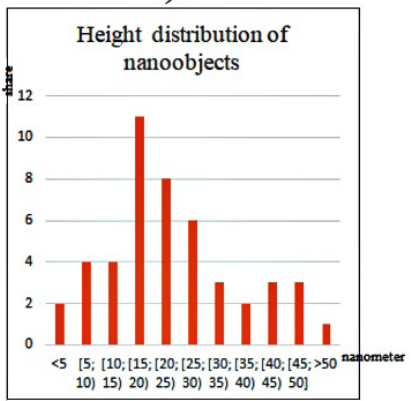

Fig. 3. a) Changes in the number of NPs on the surface of $R B C s$ during $R T ; b)$ distribution of the diameter and height (c) of NPs on the RBC surface of patients.

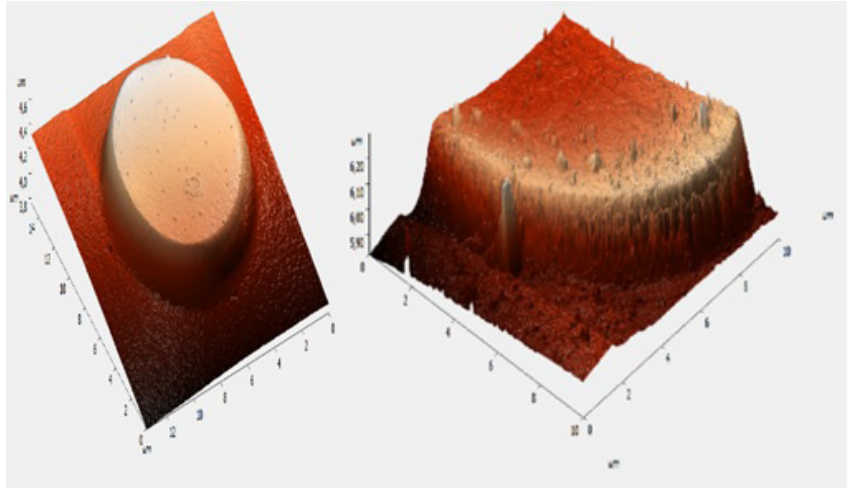

Fig. 4. AFM image of RBCs of a healthy (a) and sick (b) person.

The present study shows that RT results in morphological abnormalities and agglutination in RBCs. This is in line with previous findings that gamma radiation increases the relative number of echinocytes, sphero-echinocytes and other types of poikilocytes in a dose-dependent manner. ${ }^{(20)}$ The authors suggested that such changes in RBC morphology could stem from protein structure modifications, changes in deformability and membrane permeability as well as membrane rupture induced by radiation. ${ }^{(20)}$ In a study of rat blood following gamma irradiation treatment, an increase in RBC size and distribution width was observed, which could point to the possible radiation-induced morphological changes. ${ }^{(21)}$

Our study has also found elevated levels of NPs in CC patients' blood. The observed diameter values for NPs $(69.91 \pm 12.15 \mathrm{~nm})$ bound to RBC surface fall within the typical range for exosomes of $30 \mathrm{~nm}$ to $100 \mathrm{~nm} \cdot{ }^{(22)}$ Here we propose that these RBC-bound NPs could be EVs of various biogenesis as multiple reports have shown that elevated levels of EVs are typical for oncological conditions ${ }^{(4,10)}$ and that a significant proportion of them exist in a blood cell-bound state. ${ }^{(18)}$ One possible source of these EVs could be the CC cells, which are known to secrete abundant exosomes. ${ }^{(5)}$ These exosomes contain abnormally high levels of microRNA-2 1 and microRNA-146a associated with CC tumorigenesis, as compared to cancer-free cells. ${ }^{(5)}$ It appears that HPVs, which are the main reported cause of $\mathrm{CC}$, affect the miRNA composition of cancer exosomes. The study by Honegger et al. ${ }^{(13)}$ confirmed that the silencing of viral E6/E7 oncogenes approximately doubled the relative percentage of miRNAs inside exosomes in relation to other small RNA fractions. Interestingly, however, another study found no small RNA sequences corresponding to HPV in HPV-infected cervical carcinoma cell lines. ${ }^{(23)}$ This data points to the fact that while the link between HPV and exosomal content of CC cells cannot be denied, further research is required to determine the precise mechanisms of exosomal sorting for HPV-infected cancer cells.

It should be noted that a study by Mata-Rocha et al. found that not only exosomes derived from HeLa cells contained viral HPV DNA but also non-cancerous HPV-positive cervical samples with and without low-grade squamous intraepithelial lesions had HPV DNA (including the E6 and E7 oncogenes). ${ }^{(24)}$ These findings point to another potential source of RBC-bound EVs in CC patients: cancer-free, HPV-infected cells. While it is 
likely that RBC-bound EVs in cervical oncology patients could be attributed to cancer cells or HPV-infected cells the possibility of them being of RBC origin cannot be ruled out, especially since the average diameter of discovered EVs $(69.91 \pm 12.15 \mathrm{~nm})$ closely matches that of RBC exosomes reported by Huang et al. to be $64.08 \mathrm{~nm} .{ }^{(25)} \mathrm{RBCs}$ release vesicles into the extracellular space in the course of their normal lifespan, increasing their vesiculation levels in pathology. ${ }^{(26)}$ Their role in pathological conditions, including $\mathrm{CC}$, is under-documented; however, they have been shown to modulate immune response by raising the proliferation of $\mathrm{T}$ cells in an antigen-presenting, cell-dependent manner. ${ }^{(27)}$ In fact, depending on storage day the numbers of $\mathrm{T}$ cells were augmented by more than $50 \%$ in comparison to phytohemagglutinin stimulation alone.(27) In addition, RBCderived EVs could be involved in inflammation processes as in a study by Danesh et al. ${ }^{(27)}$ they caused significant upregulation of 14 proinflammatory cytokines in peripheral blood mononuclear cells.

In the present study, an increase in the size of RBC-bound NPs was observed in the course of RT; however, reports on the effect of irradiation on EV size present conflicting evidence. A recent study found that RT had a significant impact on exosome size, increasing it by approximately $37 \%$ at $10 \mathrm{~Gy} .{ }^{(28)}$ However, other research groups have reported no change in exosome size post-irradiation ${ }^{(16,29)}$ or an insignificant increase of around $5 \% .{ }^{(14)}$ It has been hypothesised that the alterations in exosome size may stem from the impact of radiation on multivesicular body cargo sorting pathways; ${ }^{(28)}$ however, the evidence to support this hypothesis remains limited.

The elevated abundance of NPs detected in the patients during radiotherapy is consistent with the findings that irradiated cells tend to increase their EV secretion. ${ }^{(14-17)}$ Enhanced exosome release after irradiation could be the result of upregulation of certain genes. One study found significantly elevated levels of Rab11, Rab27a, Rab27b, TSAP6, CD63, and Alix transcripts in irradiated cells, compared to controls. ${ }^{(28)}$ These genes are thought to be responsible for exosome secretion, and the effect of radiation on their abundance was dose-dependent, reaching its peak at $10 \mathrm{~Gy} .{ }^{(16)}$ While the study by Jabbari et al. ${ }^{(28)}$ investigated breast cancer, it has also been observed that exosomes specifically increased secretion of EVs post-irradiation in normal astrocytes, ${ }^{(15)}$ indicating that similar genetic changes may take place in healthy cells. These findings are consistent with our assumption that RBC-bound EVs could originate not only from CC cells but also from non-malignant HPV-infected cells or RBCs.

In conclusion, using SEM and AFM, our results reveal that CC patients exhibit changes in morphology of RBCs and in the number and size of NPs found on their surface during different stages of RT. Ionising radiation was found to influence the size and morphology of RBCs. The average value of the diameter of red blood cells in CC patients was higher than in the control group. The overall quantity of poikilocytes increased approximately $1 \%$ at each stage of therapy. The ratio of poikilocytes also varied according to the RT stage. In addition, elevated levels of NPs were found in CC patients' blood, and the quantity of NPs increased with each stage of RT. Once RT was finished the number of NPs dropped. SEM images before and after RT revealed RBC agglutination The changes observed could serve as the basis for developing efficacy indicators of cancer radiation therapy.

In addition, further research is needed to develop a precise explanation of the origin of NPs of various sizes observed on the surface of RBCs, the number of which varies during RT.

\section{Acknowledgments}

This study was supported by "The Fund of target capital management North-Eastern Federal University named after M. K. Ammosov"; Michael Ruzhansky and Junqing Huang were supported by FWO Odysseus 1 grant G.0H94.18N and Georgy V. Maksimov acknowledge financial support from Russian Sciences Foundation (grant RSF 19-79-30062) that was used for the AFM experiments.

\section{Competing Interests}

The authors declare that they have no competing interests.

\section{References}

1. WHO. Cancer. Cervical cancer. Available at: https://www. who.int/cancer/prevention/diagnosis-screening/cervicalcancer/en/

2. Ault KA. Epidemiology and natural history of human papillomavirus infections in the female genital tract. Infect Dis Obstet Gynecol. 2006;2006 Suppl: 40470.

3. Denny L. Cervical cancer: prevention and treatment. Discov Med. 2012;14(75):125-131.

4. Kim HK, Song KS, Park YS, Kang YH, Lee YJ, Lee KR, et al. Elevated levels of circulating platelet microparticles: VEGF, IL-6 and RANTES in patients with gastric cancer: possible role of a metastasis predictor. Eur $\mathrm{J}$ Cancer. 2003;39(2):184-191.

5. Liu J, Hong S, Wang X, Yu Q, Li S, Yu X, et al. Increased exosomal microRNA-21 and microRNA-146a levels in the cervicovaginal lavage specimens of patients with cervical cancer. Int J Mol Sci. 2014;15(1):758-73. doi: 10.3390/ ijms 15010758 .

6. Jang SC, Kim SR, Yoon YJ, Park KS, Kim JH, Lee J, et al. In vivo kinetic biodistribution of nano-sized outer membrane vesicles derived from bacteria. Small. 2015;11(4):456-61. doi: 10.1002/smll.201401803.

7. EL Andaloussi S, Mäger I, Breakefield XO, Wood MJ. Extracellular vesicles: biology and emerging therapeutic opportunities. Nat Rev Drug Discov. 2013;12(5):347-57. doi: $10.1038 / \mathrm{nrd} 3978$.

8. Mangino G, Chiantore MV, Luliano M, Capriotti L, Accardi L, Bonito PD, et al. Role of Extracellular Vesicles in Human Papillomavirus-Induced Tumorigenesis: in Saxena, S. K. (ed.) Current Perspectives in Human Papillomavirus. IntechOpen. 2018 Nov 9;5:1-21. doi: 10.5772/intechopen.80654.

9. Taylor DD and Gercel-Taylor C. MicroRNA signatures of tumor-derived exosomes as diagnostic biomarkers of ovarian

*Corresponding author: Irina V. Kononova, PhD. Yakut Scientific Center of Complex Medical Problems, Yakutsk, Russia. E-mail: irinakon.07@mail.ru 
cancer. Gynecol Oncol. 2008;110(1):13-21. doi: 10.1016/j. ygyno.2008.04.033.

10. Keller S, Konig AK, Marme F, Runz S, WolterinknS, Koensgen D, et al. Systemic presence and tumor-growth promoting effect of ovarian carcinoma released exosomes. Cancer Lett. 2009; 278(1):73-81. doi: 10.1016/j.canlet.2008.12.028.

11. Chai H, Brown RE. Field effect in cancer-an update. Ann Clin Lab Sci. 2009;39(4):331-7.

12. Honegger A, Schilling D, Bastian S, Sponagel J, Kuryshev V, Sultmann H, et al. Dependence of Intracellular and Exosomal microRNAs on Viral E6/E7 Oncogene Expression in HPV-positive tumor cells. PLoS Pathog. 2015 Mar 11;11(3):e1004712. doi: 10.1371/journal.ppat.1004712.

13. Li H, Chi X, Li R, Ouyang J, Chen Y. HIV-1-infected cell-derived exosomes promote the growth and progression of cervical cancer. Int J Biol Sci. 2019;15(11):2438-2447. doi: 10.7150/ijbs.38146.

14. Al-Mayah A, Bright S, Chapman K, Irons S, Carter D Goodwin E, et al. The non-targeted effects of radiation are perpetuated by exosomes. Mutat Res. 2015;772:38-45. doi: 10.1016/j.mrfmmm.2014.12.007.

15. Arscott WT, Tandle AT, Zhao S, Shabason JE, Gordon $\mathrm{IK}$, Schlaff CD, et al. Ionizing radiation and glioblastoma exosomes. Implications in tumor biology and cell migration. Transl Oncol. 2013; 6(6):638-48.

16. Mutschelknaus L, Peters C, Winkler K, Yentrapalli $\mathrm{R}$, Heider T,Atkinson MJ, et al. Exosomes derived from squamous head and neck cancer promote cell survival after ionizing radiation. PLoS One. 2016 Mar 23;11(3):e0152213. doi: 10.1371 /journal.pone.0152213.

17. Mutschelknaus L,Azimzadeh O, Heider T, Winkler K, Vetter M, Kell R, et al. Radiation alters the cargo of exosomes released from squamous head and neck cancer cells to promote migration of recipient cells. Sci Rep. 2017 Sep 29;7(1):12423. doi: 10.1038/s41598-017-12403-6.

18. Tamkovich S, Tutanov O, Efimenko A, Grigor'eva A, Ryabchikova E, Kirushina N, et al. Blood Circulating Exosomes Contain Distinguishable Fractions of Free and CellSurface-Associated Vesicles. Curr Mol Med. 2019;19(4):273285. doi: 10.2174/1566524019666190314120532.

19. Huang J, Ruzhansky M, Feng H, Zheng L, Huang X, Wang H. Feature extraction for license plate location based on L0-norm smoothing. Open Comput Sci. 2019;9(1):28-135. doi: 10.1515/comp-2019-0007
20. Xu D, Peng M, Zhang Z, Dong G, Zhang Y, Yu H. Study of damage to red blood cells exposed to different doses of $\gamma$-ray irradiation. Blood Transfus. 2012;10(3):321-30. doi: 10.2450/2012.0076-11.

21. Abdelhalim MA, Al-Ayed MS, Moussa SA, Abd Al-Sheri Ael-H, K. The effects of gamma-radiation on red blood cell corpuscles and dimensional properties in rats. Pak J Pharm Sci. 2015;28(5 Suppl):1819-22.

22. Minciacchi VR, Freeman MR, Di Vizio D. Extracellular Vesicles in Cancer: Exosomes, Microvesicles and the Emerging Role of Large Oncosomes. Semin Cell Dev Biol. 2015;40:41-51. doi: 10.1016/j.semcdb.2015.02.010.

23. Lui WO, Pourmand N, Patterson BK, Fire A. Patterns of known and novel small RNAs in human cervical cancer. Cancer Research. 2007;67(13):6031-43.

24. Mata-Rocha M, Rodríguez-Hernández RM, ChávezOlmos P, Garrido E, Robles-Vázquez C, Aguilar-Ruiz S, et al. Presence of HPV DNA in extracellular vesicles from HeLA cells and cervical samples. Enferm Infecc Microbiol Clín. 2019 Aug 5; pii:S0213-005X(19)30207-1. doi: 10.1016/j. eimc.2019.06.011. [Article in English, Spanish].

25. Huang H, Zhu J, Fan L, Lin Q, Fu D, Wei B, et al. MicroRNA Profiling of Exosomes Derived from Red Blood Cell Units: Implications in Transfusion-Related Immunomodulation. Biomed Res Int. 2019 Jun 13;2019:2045915. doi: 10.1155/2019/2045915. 26. Harisa GI, Badran MM and Alanazi FK. Erythrocyte nanovesicles: Biogenesis, biological roles and therapeutic approach: Erythrocyte nanovesicles. Saudi Pharm J. 2017;25(1):8-17. doi: 10.1016/j.jsps.2015.06.010.

27. Danesh A, Inglis HC, Jackman RP, Wu S, Deng X, Muench MO. Exosomes from red blood cell units bind to monocytes and induce proinflammatory cytokines, boosting T-cell responses in vitro. Blood. 2014;123(5):687-96. doi: 10.1182/blood-2013-10-530469.

28. Jabbari N, Nawaz $M$ and Rezaie J. Ionizing radiation increases the activity of exosomal secretory pathway in MCF7 human breast cancer cells: A possible way to communicate resistance against radiotherapy. Int J Mol Sci. 2019 Jul 25;20(15). pii: E3649. doi: 10.3390/ijms20153649.

29. Bagheri HS, Mousavi M, Rezabakhsh A, Rezaie J, Rasta SH, Nourazarian A, et al. Low-level laser irradiation at a high power intensity increased human endothelial cell exosome secretion via Wnt signaling. Lasers Med Sci. 2018;33(5):11311145. doi: 10.1007/s10103-018-2495-8. 